\title{
EALTERIDAD Diagnóstico comunitario ante desastres climáticos: Una experiencia de aprendizaje-servicio
}

\section{Community assessment in the face of climatic disasters: A Service-Learning experience}

(D) Dr. José Sandoval-Díaz ${ }^{1}$ es docente e investigador de la Universidad del Bío Bío (Chile) (jsandoval@ubiobio.cl) (https://orcid.org/0000-0001-7247-7113)

(D) David Cuadra-Martínez es docente e investigador de la Universidad de Atacama (Chile) (david.cuadra@uda.cl) (https://orcid.org/0000-0002-0810-2795)

(D) Cristian Orellana-Fonseca es docente e investigador de la Universidad del Bío Bío (Chile) (corellana@ubiobio.cl) (https://orcid.org/0000-0001-8614-8011)

(D) Eduardo Sandoval-Obando es docente e investigador de la Universidad Autónoma de Chile (https://orcid.org/0000-0001-7471-6536)

Recibido: 2020-05-14 / Revisado: 2020-12-09 / Aceptado: 2020-12-10 / Publicado: 2021-01-01

\section{Resumen}

El incremento de eventos extremos - a causa del cambio climático - conlleva no solo a la necesidad local de fortalecer las capacidades de afrontamiento y de resiliencia a nivel comunitario, sino también, al replanteo sobre la idoneidad de los planes formativos universitarios en el desarrollo de profesionales competentes ante estos nuevos riesgos emergentes. Bajo este contexto, el presente trabajo tuvo como objetivo valorar la implementación de un proceso de diagnóstico de necesidades comunitarias ante un desastre climático, esto mediante la aplicación metodológica de la estrategia de aprendizaje-servicio (ApS) en un curso de estudiantes de psicología en Chile. Para esto seleccionamos un estudio de caso representativo de gestión comunitaria ante un desastre climático en el norte de Chile, esto bajo un diseño de investigación acción-participativa (IAP). Los datos son producidos mediante la triangulación intermétodo de seis técnicas, esquematizando los resultados del proceso de aprendizaje en cuatro áreas: i) teórico-conceptual, ii) metodológica, iii) práctica y iv) ético-política. Los resultados relevan el uso de ApS en el proceso de enseñanza-aprendizaje del diagnóstico comunitario, identificando tanto ventajas como limitaciones en el desarrollo de competencias disciplinares, transversales profesionales y del servicio entregado. Se concluye con la importancia de integrar estrategias horizontales, participativas y situadas en los procesos de enseñanza profesional, esto acorde al tipo de formación universitaria integral que requiere la problemática psicosocial compleja del cambio climático.

Descriptores: Aprendizaje-servicio, diagnóstico comunitario, formación profesional, investigación acción participativa, cambio climático, Chile.

\begin{abstract}
The increase of extreme events caused by climate change has lead no only to the local need for strengthen the coping and resilience capacity at a community level, but also, to rethink about the suitability of the university study programs in order to develop competent professionals in the face of these new emerging risks. In this context, the objective of this study was to assess the implementation of a community needs assessment process before a climate disaster through the methodological implementation of the Service-Learning strategy (ApS) in a course of psychology students in Chile. To do so, we selected a representative case study of community management in the face of a climate disaster in the north of Chile, using a participatory action research design (PAR). The data were produced using an inter-method triangulation of six techniques, creating a four areas scheme of the learning process results. These areas were: i) theoretical-conceptual, ii) methodological, iii) practical and iv) ethical-political.The results showed the use of ApS in the teaching-learning process of the community assessment, identifying both advantages and limitations in the development of disciplinary, transversal and professional competences, as well as those competences related to the service provided. This study concludes that it is important to integrate horizontal, participative strategies, and use them in the process of professional training and education, according to the integral type of university education required to face the complex psychosocial problem of climate change.

Keywords: Service-learning, community assessment, professional training, participatory action research, climate change, Chile.
\end{abstract}

Forma sugerida de citar: Sandoval-Díaz, J., Cuadra-Martínez, D., Orellana-Fonseca, C., \& Sandoval-Obando, E. (2021). Diagnóstico comunitario ante desastres climáticos: Una experiencia de aprendizaje-servicio. Alteridad, 16(1), 23-37. https://doi.org/10.17163/alt.v16n1.2021.02 


\section{El cambio climático y rol formativo universitario}

La adaptación al cambio climático se ha convertido en uno de los principales objetivos para el Desarrollo sostenible a escala global (Intergovernmental Panel on Climate Change [IPCC], 2014). En América Latina, el cambio ambiental global no solo ha conllevado la emergencia de nuevos riesgos socionaturales, tales como (i) pérdida de biodiversidad, (ii) subida del nivel del mar, (ii) olas de calor y (iv) aumento de peligros hidrometeorológicos, como sequías e inundaciones; sino que, a su vez, ha amplificado e intensificado la vulnerabilidad social de los territorios (Griselda-Günther \& Gutiérrez, 2017; Sánchez \& Reyes, 2015).

En términos de impacto, solo considerando el año 2018, los eventos hidrometeorológicos afectaron a un total de 57,3 millones de personas, siendo liderado por las inundaciones, con un promedio de 35,4 millones de afectados (UN Office for Disaster Risk Reduction [UNDRR], 2019).

Estos intempestivos eventos, ha conllevado la reconfiguración de las estrategias gubernamentales de reducción del riesgo desastre (RRD), las cuales han desplazado el foco desde la respuesta institucional hacia el fortalecimiento de capacidades locales y de resiliencia comunitaria (Gaillard et al., 2019; Sandoval-Díaz, 2020).

En relación con esto, el artículo seis de la Convención Marco de las Naciones Unidas sobre el Cambio Climático (CMNUCC) releva el papel de la educación, formación y sensibilización ante este riesgo, requiriendo no solo su integración curricular, sino repensar el rol de la educación ante la "alfabetización climática" (IPCC, 2014). De acuerdo con esto, paulatinamente, diversos países han ido incorporando contenidos curriculares, tanto en educación formal primaria y/o secundaria (United Nations Educational, Scientific, and Cultural Organization [UNESCO], 2015). En tanto, si bien a nivel universitario esta problemática se encuentra en expansión, predominantemente en disciplinas y posgrados vinculados a ciencias ambientales, es en el campo profesional de las ciencias sociales donde se requiere de colaboradores comprometidos para fortalecer capacidades adaptativas de resiliencia comunitaria (García-Lirios et al., 2014; Ricardo et al., 2019).

Por lo tanto, es importante repensar el rol educativo de las universidades ante estas problemáticas emergentes, con el objetivo de que no solo se ocupen de su desarrollo investigativo básico, sino también, en la facilitación de espacios formativos aplicados que contribuyan al desarrollo de profesionales competentes ante el diagnóstico e intervención de estas problemáticas (De Castro \& Domínguez, 2018).

Para el caso de la formación profesional en Chile, ser competente a nivel institucional se define como:

La capacidad de un individuo para movilizar, tanto sus recursos internos (conocimientos, habilidades y actitudes), como aquellos externos disponibles en el entorno de su área de desempeño, para solucionar problemas complejos que se presenten en el desarrollo de su profesión o actividad. (Comisión Nacional de Acreditación de Chile [CNA], 2015 p. 4)

En el campo formativo universitario, y en las ciencias humanas en particular, un recurrente problema son las dificultades de los estudiantes para transferir, generalizar y aplicar los contenidos teóricos aprendidos al interior de las aulas, hacia situaciones prácticas reales (BarrónTirado, 2009; Cuadra et al., 2018), conllevando no solo dificultades en el cómo proceder operativamente ante contextos diferenciados, sino también, en el cómo favorecer la implicación y participación activa por parte de las propias comunidades involucradas (Stringer, 2008).

A su vez, esta dificultad en la transferencia, no se remite solo a las asignaturas que tributan a los ámbitos de potencial desempeño profesional — sino también - en aquellas asignaturas formativas vinculadas a la reflexividad y metodologías investigativas-científicas (Bassi, 2015; 
Orellana-Fonseca et al., 2019). Desde el imaginario universitario aquellas asignaturas de carácter investigativo solo adquieren "sentido" ante el potencial desarrollo de la tesis de grado o para la escritura de "papers" (si es que los estudiantes presentan interés por el ámbito académicouniversitario), ignorando muchas veces (al igual que sus profesores), las potenciales ventajas que esta forma de racionalidad científica entrega ante situaciones de desempeño profesional concreto, tales como son los procesos de diagnóstico e intervención social (Muñoz-Arce et al., 2017).

Estos procesos de diagnóstico e intervención social, desde una perspectiva transdiciplinaria, tienen como objetivo no solo (i) delimitar y evaluar necesidades psicosociales ante una problemática particular, para posteriormente (ii) desplegar acciones fundamentadas para solucionar dicha problemática, sino también, (iii) posibilitar espacios inclusivos y colaborativos con y desde las propias comunidades implicadas, esto con el objetivo de mediatizar la adquisición reflexiva de capacidades transformadoras de los contextos vulnerados (Freire, 2012; Ortega, 2015).

En línea con esto último, sostenemos que la formación de competencias ante el diagnóstico de problemáticas sociales deben superar el binarismo conocer-hacer de la formación disciplinariaprofesional convencional (Matus, 2002), integrando sostenidamente estrategias y procedimientos indagatorios dialogantes situados, que consideren no solo las competencias técnicas sino también, la subjetividad reflexiva y lógica subyacente al quehacer investigativo como práctica transdisciplinar transformadora (Cuadra-Martínez et al., 2018; Muñoz-Arce et al. 2017; Ortega, 2015), esto acorde a una formación universitaria integral de competencias profesionales y existenciales ante la actual emergencia de problemáticas complejas a escala global (Morin, 2016).

En función de esto, relevamos el papel práctico y heurístico del proceso de investigación aplicado al campo profesional, comprendido como una potencial caja de herramientas para fundamentar reflexivamente decisiones de diagnóstico e intervención social (Bassi, 2015; Orellana-Fonseca et al. 2019), más en los casos donde se torna necesario el levantamiento y análisis de datos de problemáticas complejas, como el cambio climático (García-Lirios et al., 2014; Ricardo et al., 2019).

En síntesis, este desarrollo formativo de competencias investigativas aplicadas al ámbito profesional nos lleva a replantear las formas convencionales de enseñanza-aprendizaje, incitándonos a la incorporación de metodologías participativas e implicativas-activas, que no solo tributen al buen desempeño "declarativo teórico", sino también a su potencial aplicación reflexiva en contextos problemáticos situados (López-Noguero, 2017). De acuerdo con esto, una potencial estrategia formativa para este cometido es el aprendizaje-servicio (ApS).

\subsection{El aprendizaje-servicio}

La formación profesional requiere de espacios educativos que logren trasferir el conocimiento desde el aula hacia la realidad concreta, esto bajo la adquisición y desarrollo de competencias transversales $^{2}$ y disciplinares (Wendler, 2012; Cuadra-Martínez et al., 2018). No obstante, bajo este nuevo contexto de incertidumbre, se sostiene la necesaria implementación de metodologías experienciales-activas, por sobre el mero aprendizaje reproductivo sin valor de uso, requiriendo la mixtura de la reflexión del aprender haciendo constante (Dewey, 1985), y el compromiso social activo con las necesidades vitales de las comunidades más desposeídas (Freire, 2012).

Es así como la metodología del ApS permitiría desarrollar competencias, transferir conocimientos a las comunidades, educar en valores, desarrollar un mayor autoconocimiento, un fuerte sentido de responsabilidad social y de compromiso ciudadano (Rodríguez, 2014), esto bajo el contexto actual que desafía cada vez más a los profesionales (Pizarro \& Hasbún, 2019). A su vez, esta propuesta articula simultáneamente la responsabilidad socioeducativa, la innovación 
pedagógica y el activo protagonismo estudiantil, tanto en el territorio a intervenir, como en los propios procesos de enseñanza-aprendizaje (Mayor, 2019). En la actualidad existen más de 147 nociones de ApS, las cuales se materializan en (i) variados contextos sociocultuales, (ii) instituciones con distinta dependencia administrativa, (iii) intersectorialidad en cuanto al tipo de servicio, (iv) implementación curricular variada, (v) disciplinas distintas, (vi) múltiples niveles y edades de sus participantes (Mayor, 2019).

Para Furco (2011), uno de los principales autores del área, el ApS es "una pedagogía de enseñanza por la que los estudiantes adquieren una mejor comprensión del contenido académico, aplicando competencias y conocimientos para el beneficio de la sociedad" (p. 23). Por otro lado, en términos aplicados, la Red Nacional de Aprendizaje Servicio de Chile (REASE), creada el 2011, lo concibe como:

Un enfoque pedagógico de enseñanza-aprendizaje aplicado en cursos, prácticas y tesis, utilizado para la resolución de problemáticas sociales reales y sentidas, mediante un servicio de calidad, en el que de manera integrada y colaborativa los tres actores centrales del proceso (docentes, estudiantes y socios comunitarios) se vinculan y trabajan en conjunto. (Claire et al., 2019 p. 38)

Si bien existen dificultades para homogenizar esta práctica educativa bajo una misma expresión, existe cierto consenso en tres criterios fundamentales: (i) Servicio comunitario con el objetivo de responder a problemáticas sociales, (ii) pedagogía activa que requiere el protagonismo del estudiantado y cuerpo académico, y (iii) planificación integrada de contenidos curriculares con actividades de servicio comunitario que posibiliten la adquisición de competencias (Montes, Tapia \& Yaber, 2011). Respecto a la arquitectura pedagógica de la ApS se han identificado cinco dimensiones centrales: i) aprendizaje, ii) servicio, iii) participación, iv) actividad de utilidad social y v) reflexión, las cuales, si bien no se expresan de igual manera y énfasis, manifiestan un estado final deseable (Mayor, 2009).

De acuerdo con estos antecedentes, el presente trabajo tiene como objetivo general valorar la implementación de un proceso de diagnóstico de necesidades comunitarias ante un desastre climático, esto mediante la aplicación metodológica de ApS en estudiantes universitarios de psicología en Chile. Como objetivos específicos de aprendizaje buscamos: i) Identificar las ventajas de aprendizaje en las áreas teórico-conceptuales, metodológicas, prácticas y ética-políticas del diagnóstico de necesidades comunitarias bajo el ApS, ii) Identificar las limitaciones de aprendizaje en las cuatro áreas mencionadas anteriormente; mientras que para el ámbito de servicios se busca iii) Generar un diagnostico participativo de necesidades psicosociales ante el desastre.

\subsection{Características del caso de estudio}

La carrera de psicología de la Universidad de Atacama (UDA) surge con el objetivo de iniciar la formación regional de profesionales cuyas competencias científicas-prácticas son el diagnóstico, la evaluación y la intervención en procesos que involucran a personas, grupos y organizaciones, con capacidad de discernimiento ético $y$ respeto por la naturaleza humana en el ejercicio de la profesión (Departamento de Psicología UDA, 2015).

En línea con el modelo educativo universitario, de corte constructivista, y en concordancia con la propuesta 2008-2011 de homologación de competencias curriculares de psicólogos por parte de la red de universidades estatales de Chile, ${ }^{3}$ esta carrera enfatiza la formación de competencias transversales y específicas en diez semestres (Juliá, 2013).

Es así cómo esta formación por competencias incorpora a lo largo de su currículo cuatro asignaturas de taller de integración práctica-profesional: i) acercamiento al campo profesional (segundo semestre); ii) áreas de desa- 
rrollo e inserción profesional (cuarto semestre); iii) diagnóstico psicosocial (sexto semestre) e iv) intervención psicosocial (octavo semestre), esto como previas inducciones a la práctica profesional final (Departamento de Psicología UDA, s.f.).

Bajo este marco formativo, se decide incorporar ApS a la asignatura taller de integración III (diagnóstico psicosocial), esto a partir de la inquietud de un grupo de académicos, quienes, a raíz de un desastre climático ocurrido el 2015 (ver apartado 1.4), buscan no solo responder a la solicitud de apoyo de una comunidad afectada, sino también, reforzar la comprensión aplicada de algunas herramientas metodológicas de investigación previamente enseñadas de forma teórica.

Atendiendo a lo anterior, previo al inicio del segundo semestre del año 2016, se diseña y planifica la incorporación de la estrategia de ApS al taller de integración III, ante lo que se inicia la búsqueda de colaboradores territoriales llegando a la junta vecinal $\mathrm{N}^{\circ} 36$ de la localidad de Paipote, quienes se encontraban en pleno diseño del plan de reconstrucción habitacional posdesastre. En función de esto, se realizaron una serie de reuniones entre profesores y dirigentes comunitarios para aunar objetivos recíprocos y plausibles a desarrollar, donde se consensuó desarrollar un diagnóstico de necesidades comunitarias.

En el presente trabajo entendemos diagnóstico de necesidades como el proceso de elaboración y sistematización de información que permite conocer la problemática y requisitos psicosociales dentro de un contexto, permitiendo jerarquizar necesidades, y como consecuencia, potenciales estrategias de intervención procurando dar viabilidad para su implementación (Aguilar-Idañez \& Ander-Egg, 2001). Los requisitos que debe cumplir este tipo de diagnóstico es que debe ser amplio (para esto se incorporan distintas estrategias de investigación cualitativas y cuantitativas), en lenguaje sencillo, preciso y oportuno, para el presente caso la potencial reconstrucción habitacional posdesastre.

\subsection{El desastre climático en Atacama}

Un evento hidrometeorológico extremo, el 25 de marzo del 2015 (25M), producto de una baja segregada asociada a precipitaciones, causó lluvias torrenciales en 17 quebradas en Atacama, Chile. En Copiapó, capital regional, las lluvias arrastraron gran cantidad de agua enterrando la ciudad bajo una capa de sedimentos de $31 \mathrm{~cm}$ de espesor, dejando a más del 50\% de las viviendas sin sistema de alcantarillado. En cuanto a la afectación, se registraron 22 personas fallecidas, 28000 damnificados, 2000 viviendas destruidas y 5000 con daño mayor, conllevando un impacto económico superior a 46 millones de USD (Izquierdo et al., 2018).

En términos de impacto psicosocial los grupos expuestos-susceptibles que presentaron una significativa severidad subjetiva fueron los adulto/as mayores, personas con discapacidad/ enfermedad crónica, de sexo femenino, con bajos ingresos económicos y sin educación superior, sumándose quienes se encuentran en la condición de daño parcial-total en su vivienda y no recibieron apoyo para la reconstrucción habitacional (Sandoval-Díaz \& Cuadra-Martínez, 2020).

En términos físicos-materiales, la altura media de afectación fue de $45 \mathrm{~cm}$, identificándose cuatro sectores de la ciudad en los que la inundación superó el metro de lámina de agua, siendo uno de ellos la localidad de Paipote, zona cero del desastre. De acuerdo con Izquierdo et al. (2018), las inundaciones causadas por flujos de derrubios y fango constituyen un riesgo a escala global, especialmente en zonas de grandes desniveles y clima árido, como es el caso de Paipote (Ver figura 1). Esta localidad residencial de 20 000 habitantes, fundada en 1913, se ubica entre la conurbación de las comunas de Tierra Amarilla y Copiapó, a 8 kilómetros de esta última. 
Figura 1. Diagrama aéreo del caso de estudio

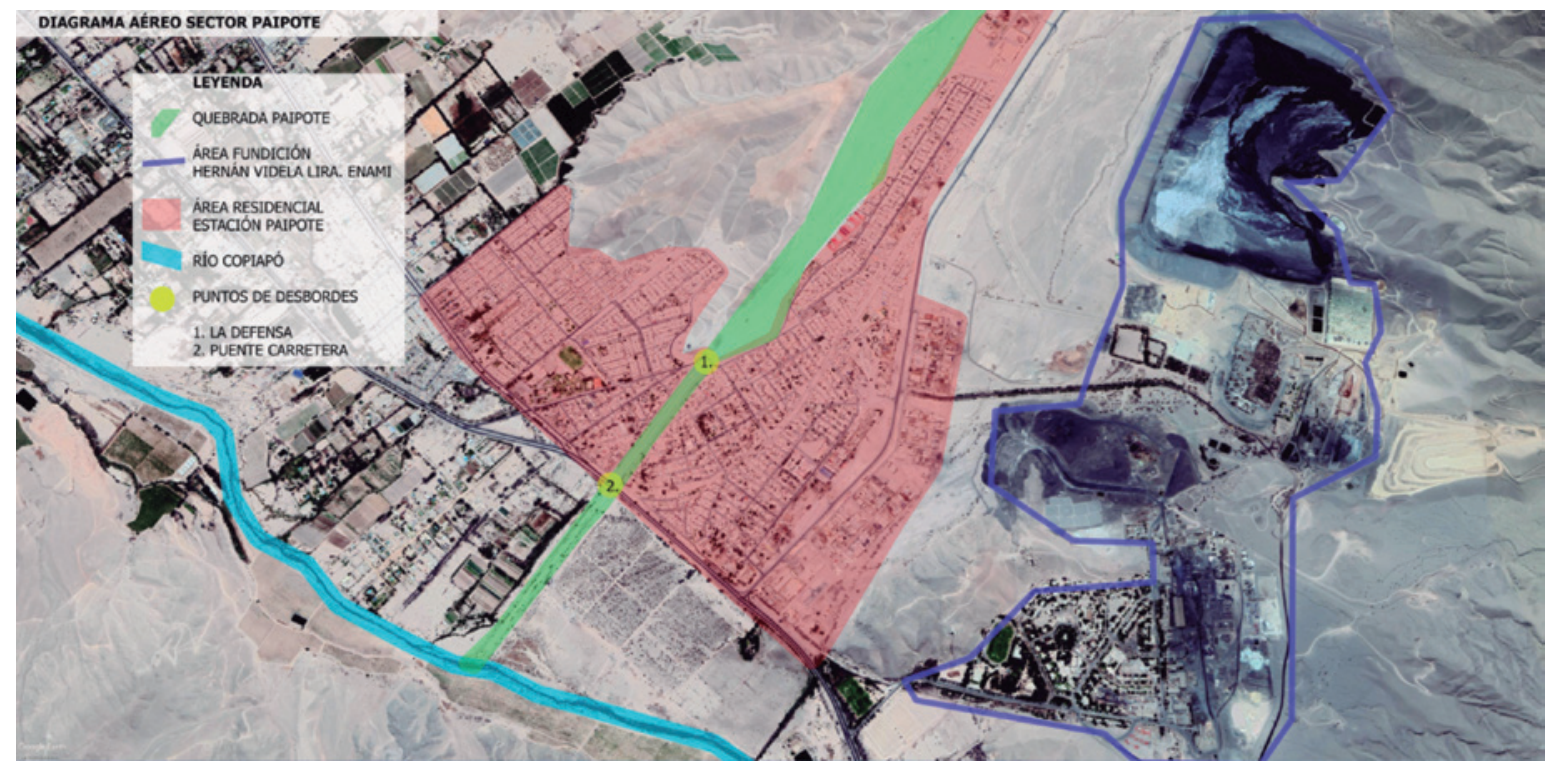

Fuente: Astudillo-Pizarro y Sandoval-Díaz (2019, p. 315).

A fines del año 2016, los dirigentes vecinales de la junta vecinal $\mathrm{N}^{\circ} 36$ de Paipote intentan organizarse para acelerar la lenta reconstrucción de sus barrios y viviendas devastados por el desastre (ocurrido hace más de un año y medio), así como también para solicitar apoyo a actores tanto gubernamentales como de la sociedad civil, como fue el caso del Departamento de psicología de la UDA. Ante esto, un grupo de docentes diseñaron un programa formativo en diagnóstico de necesidades psicosociales a nivel comunitario. La implementación de dicho programa se realizó con la finalidad de responder a dos objetivos: levantar un diagnóstico en conjunto con la junta vecinal, y fortalecer herramientas de formación profesional de estudiantes de octavo semestre.

\section{Metodología}

Se utilizó un estudio de caso ${ }^{4}$ de gestión comunitaria ante un desastre climático, para esto se seleccionó intencionadamente la localidad de Paipote de Atacama como un caso representativo (Gerring, 2007). Se implementó un diseño de Investigación Acción Participativa [IAP], con el objetivo de enfatizar la participación y acción de la comunidad afectada (Stringer, 2008). Fundamental a la IAP es la fase de sensibilización, involucramiento y producción de información, vinculada a la experiencia participativa directa de los profesores responsables del curso a lo largo del ciclo del desastre, alternando instancias de colaboración con la comunidad por medio de la participación en asambleas intersectoriales vinculadas a la reconstrucción habitacional y organización de actividades recreativas en conjunto. Por último, con el fin de que los estudiantes aplicaran técnicas investigativas enseñadas en asignaturas previas, se incorporó la "triangulación de métodos"5 como criterio de validación cruzada (Flick, 2014), esto con el objetivo que utilizaran tanto estrategias cuantitativas como cualitativas (CUAN-CUAL) de producción de datos orales, escritos y visuales, las cuales serán descritas en el apartado de técnicas (ver imagen 1).

Respecto a los socios colaboradores, se utilizó un muestreo intencionado a escala vecinal, procediendo "según la relevancia de los casos, en lugar de hacerlo por su representatividad 
cuantitativa" (Flick, 2007, p. 80). Con base en lo presentado se trabajó en la junta vecinal $\mathrm{N}^{\circ} 36$ de Paipote, asentada en la zona cero del desastre, la que contaba con 174 familias con distintos grados de afectación habitacional.

Figura 2. Aplicación de la técnica de la deriva en la localidad de Paipote

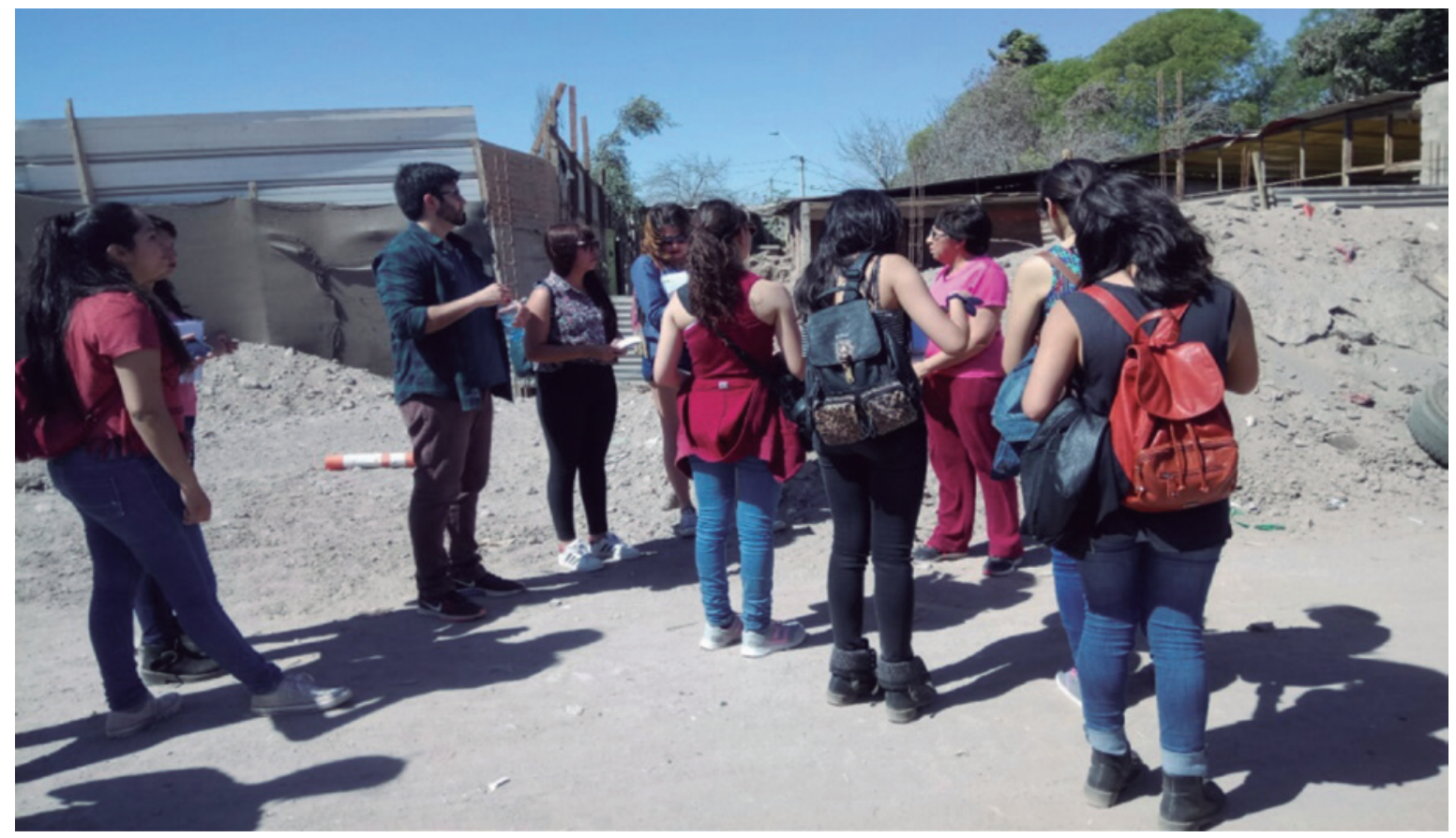

Fotografía: José Sandoval-Díaz.

\subsection{Procedimientos}

La implementación del ApS se realizó desde mediados de julio hasta fines diciembre del año 2016. El taller de integración III contaba con 34 estudiantes inscritos de octavo semestre de psicología. A nivel de servicio, previo inicio de la asignatura, se realizaron dos reuniones de coordinación con dirigentes vecinales, con el objetivo de: consensuar los productos, periodicidad y procedimientos del diagnóstico, y conocer la historia local y experiencia vinculada al desastre climático, posibilitando un primer acercamiento territorial al contexto y la problemática a abordar. Posterior a esta coordinación, junto a la informante clave (dirigente vecinal) se tomó contacto con algunas familias damnificadas, con el objetivo de difundir el potencial trabajo a realizar.
A nivel de aprendizaje, inicialmente, se establecieron sesiones teórico-prácticas semanales con una duración aproximada de una hora y media cronológica (duración del bloque de la asignatura), las que se ampliaban a tres horas si se realizaba en terreno. Para el desarrollo de la asignatura, los estudiantes se auto-agruparon por afinidades personales, (en un máximo de cuatro personas), con el objetivo de priorizar un trabajo en equipo basado en el conocimiento previo. En total se implementaron 13 sesiones (más dos sesiones recreativas junto a la comunidad), las cuales se realizaron tanto en terreno como en las aulas universitarias. Las sesiones son descritas brevemente en la siguiente tabla. 
Tabla 1. Sesiones de actividades implementadas en el taller de integración III

\begin{tabular}{|c|c|c|c|}
\hline Fecha & Actividad & $\begin{array}{l}\text { Tipo de } \\
\text { actividad }\end{array}$ & Breve descripción \\
\hline $24 / 07$ & $\begin{array}{l}\text { Reuniones de coordinación previa } \\
\text { con la comunidad. }\end{array}$ & $\begin{array}{l}\text { Práctica de } \\
\text { terreno }\end{array}$ & $\begin{array}{l}\text { Reuniones del cuerpo académico con dirigentes vecinales } \\
\text { para delimitación de objetivos y procedimientos. }\end{array}$ \\
\hline 06/08 & Diseño IAP. & Teórica & $\begin{array}{l}\text { Presentación en aula del diseño, sintetizando sus compo- } \\
\text { nentes teóricos, prácticos y procedimentales. }\end{array}$ \\
\hline $30 / 08$ & Primera inducción al campo. & $\begin{array}{l}\text { Práctica en } \\
\text { Terreno }\end{array}$ & $\begin{array}{l}\text { A nivel grupal se realizó un recorrido a pie por la locali- } \\
\text { dad, utilizando un diario de campo para anotar primeras } \\
\text { impresiones. }\end{array}$ \\
\hline $13 / 09$ & Observación guiada: la deriva. & $\begin{array}{l}\text { Práctica de } \\
\text { terreno }\end{array}$ & $\begin{array}{l}\text { Aplicación de una técnica de observación participante } \\
\text { guiada por los propios dirigentes vecinales. }\end{array}$ \\
\hline $30 / 09$ & $\begin{array}{l}\text { Primera jornada gestión local del } \\
\text { riesgo de desastre UDA. }\end{array}$ & $\begin{array}{l}\text { Seminario- } \\
\text { conversa- } \\
\text { torio }\end{array}$ & $\begin{array}{l}\text { Realizado con distintos expertos locales sobre la temática } \\
\text { de gestión de riesgo de desastre, del que participaron tan- } \\
\text { to dirigentes vecinales como estudiantes. }\end{array}$ \\
\hline $04 / 10$ & $\begin{array}{l}\text { Diagnóstico de necesidades obser- } \\
\text { vadas: estáticas y dinámicas. }\end{array}$ & Teórica & $\begin{array}{l}\text { Presentación en aula del diagnóstico de necesidades psico- } \\
\text { sociales, sintetizando sus componentes teórico-prácticos. }\end{array}$ \\
\hline $11 / 10$ & Diseño de una escala cuantitativa. & $\begin{array}{l}\text { Teórica- } \\
\text { aplicada }\end{array}$ & $\begin{array}{l}\text { En el aula los estudiantes diseñaron preguntas cerradas } \\
\text { de opción i) dicotómica, ii) politómica y de iii) escala Likert; } \\
\text { esto de acuerdo con las impresiones cualitativas recogi- } \\
\text { das en terreno. }\end{array}$ \\
\hline $25 / 10$ & Aplicación de escalas cuantitativas. & $\begin{array}{l}\text { Práctica de } \\
\text { terreno }\end{array}$ & $\begin{array}{l}\text { Aplicación en terreno de escalas psicométricas y pregun- } \\
\text { tas cerradas (consensuadas y seleccionadas en la clase } \\
\text { anterior), esto vía muestreo probabilístico de viviendas por } \\
\text { manzana. }\end{array}$ \\
\hline $08 / 11$ & $\begin{array}{l}\text { Estrategias participativas: Ecomapa } \\
\text { y cartografía social. }\end{array}$ & Teórica & $\begin{array}{l}\text { Presentación introductoria de técnicas participativas sinte- } \\
\text { tizando sus ventajas y limitaciones teórico-prácticas. }\end{array}$ \\
\hline $29 / 11$ & $\begin{array}{l}\text { Matriz de sistematización de diag- } \\
\text { nóstico de necesidades. }\end{array}$ & $\begin{array}{l}\text { Teórica- } \\
\text { aplicada }\end{array}$ & $\begin{array}{l}\text { Presentación teórica de matriz de capacidades y vulnera- } \\
\text { bilidades comunitarias (MCV). }\end{array}$ \\
\hline $06 / 12$ & $\begin{array}{l}\text { Presentación de avances grupales } \\
\text { (Matrices). }\end{array}$ & $\begin{array}{l}\text { Teórica- } \\
\text { expositiva }\end{array}$ & $\begin{array}{l}\text { Presentación grupal de avance de matriz MCV, la que fue } \\
\text { retroalimentada por docentes y pares estudiantiles. }\end{array}$ \\
\hline $13 / 12$ & Entrega de informe final de síntesis. & Teórica & $\begin{array}{l}\text { Con la retroalimentación realizada, los grupos de trabajo } \\
\text { debían elaborar un informe final. }\end{array}$ \\
\hline $\begin{array}{l}10 / 08 \\
21 / 12\end{array}$ & $\begin{array}{l}\text { Actividades recreativas: i) Celebra- } \\
\text { ción del día del niño/a y ii) Apoyo } \\
\text { celebración navidad comunitaria. }\end{array}$ & $\begin{array}{l}\text { Actividades } \\
\text { recreativas }\end{array}$ & $\begin{array}{l}\text { Realización y apoyo de dos actividades recreativas junto } \\
\text { al cuerpo académico, estudiantes y comunidad. }\end{array}$ \\
\hline
\end{tabular}

Fuente: Elaboración propia

\subsection{Técnicas de producción de datos}

Con los objetivos de a) fortalecer el aprendizaje aplicado investigativo, así como también, b) aumentar la validez del proceso de diagnóstico de necesidades, se utilizó la estrategia de triangulación de métodos CUAN-CUAL (Flick, 2014). Estas técnicas —en primer lugar- fueron enseñadas en sesiones de clase (ver tabla 1), para posteriormente, aplicarse en el trabajo de campo.
La enseñanza de estas técnicas se realizó flexiblemente, esto de acuerdo con los tiempos y características de las sesiones respectivas, considerando para esto: a) temáticas tratadas y emergentes en los terrenos, b) disposición de los estudiantes, c) características del aula y de los recursos a utilizar, por mencionar solo algunos aspectos. La tabla 2 describe las técnicas utilizadas, con su respectivo objetivo, justificación y etapa de utilización. 
Tabla 2. Descripción de las técnicas enseñadas y aplicadas por los estudiantes para el diagnóstico

\begin{tabular}{|c|c|c|c|}
\hline $\begin{array}{l}\text { Contexto de } \\
\text { uso }\end{array}$ & Técnica & Objetivo & Justificación \\
\hline Terreno práctico & $\begin{array}{l}\text { Entrevista narrati- } \\
\text { va (Flick, 2007). }\end{array}$ & $\begin{array}{l}\text { Conocer la historia local, como } \\
\text { los antecedentes y característi- } \\
\text { cas del desastre. }\end{array}$ & $\begin{array}{l}\text { Primer acercamiento cara a cara con la co- } \\
\text { munidad desde una perspectiva situada. }\end{array}$ \\
\hline Terreno práctico & $\begin{array}{l}\text { La deriva (Pellicer } \\
\text { et al., 2013). }\end{array}$ & $\begin{array}{l}\text { Explorar los significados y senti- } \\
\text { dos espaciales ante la experien- } \\
\text { cia de desastre. }\end{array}$ & $\begin{array}{l}\text { Técnica de observación guiada que terri- } \\
\text { torializó las narrativas emergentes de la } \\
\text { entrevista, esto a través de un recorrido en } \\
\text { la zona de exposición al riesgo. }\end{array}$ \\
\hline $\begin{array}{l}\text { Aplicación } \\
\text { práctica aula }\end{array}$ & $\begin{array}{l}\text { Cartografía social } \\
\text { participativa (Vé- } \\
\text { lez et al., 2012). }\end{array}$ & $\begin{array}{l}\text { Producir un mapa, a escala } \\
\text { barrial, de potenciales amena- } \\
\text { zas, vulnerabilidades y espa- } \\
\text { cios de seguridad ante riesgos } \\
\text { climatológicos. }\end{array}$ & $\begin{array}{l}\text { Técnica dialógica que grafica el uso y } \\
\text { apropiación espacial, en la que se dibujan } \\
\text { lugares expuestos-susceptibles y de segu- } \\
\text { ridad ante riesgos. }\end{array}$ \\
\hline $\begin{array}{l}\text { Aplicación } \\
\text { práctica aula }\end{array}$ & $\begin{array}{l}\text { Ecomapa (Fernán- } \\
\text { dez et al., 2011). }\end{array}$ & $\begin{array}{l}\text { Producir un mapa relacional de } \\
\text { actores sociales identificando el } \\
\text { grado de cercanía o distancia } \\
\text { percibido. }\end{array}$ & $\begin{array}{l}\text { Técnica ecológica que grafica las rela- } \\
\text { ciones de cercanía, distancia o ausencia } \\
\text { entre comunidad e instituciones públicas/ } \\
\text { privadas a lo largo del ciclo del desastre. }\end{array}$ \\
\hline $\begin{array}{l}\text { Terreno } \\
\text { práctico }\end{array}$ & $\begin{array}{l}\text { Muestreo y apli- } \\
\text { cación de escalas } \\
\text { de impacto psico- } \\
\text { social (Sandoval- } \\
\text { Díaz \& Cuadra- } \\
\text { Martínez, 2020). }\end{array}$ & $\begin{array}{l}\text { Aplicar competencias de } \\
\text { muestreo y recogida de datos } \\
\text { cuantitativos }\end{array}$ & $\begin{array}{l}\text { Los estudiantes aplicaron un cuadernillo } \\
\text { de escalas psicosociales previo entrena- } \\
\text { miento. Posteriormente analizaron los re- } \\
\text { sultados de forma general vía estadística } \\
\text { descriptiva. }\end{array}$ \\
\hline $\begin{array}{l}\text { Sistematización } \\
\text { en aula }\end{array}$ & $\begin{array}{l}\text { Matriz de capaci- } \\
\text { dades y vulnerabi- } \\
\text { lidades (Anderson } \\
\text { \& Woodrow, 1989). }\end{array}$ & $\begin{array}{l}\text { Sistematizar la información le- } \\
\text { vantada, utilizando una matriz } \\
\text { MCV }\end{array}$ & $\begin{array}{l}\text { A nivel grupal los estudiantes debieron } \\
\text { sistematizar la información levantada para } \\
\text { elaborar un diagnóstico comunitario para } \\
\text { las áreas a) físico-material, b) organizacio- } \\
\text { nal y c) motivacional. }\end{array}$ \\
\hline
\end{tabular}

Fuente: Adecuación propia de Sandoval et al. (2018).

\section{Resultados}

Los resultados se esquematizan en las tablas 3 y 4, según los objetivos específicos del estudio, identificando tanto ventajas como limitaciones de aprendizaje en las áreas i) teórico-conceptuales, ii) metodológicas, iii) prácticas y iv) ética-políticas del diagnóstico de necesidades comunitarias bajo ApS.

Tabla 3. Ventajas en la enseñanza-aprendizaje del diagnóstico psicosocial bajo ApS

\begin{tabular}{|c|c|c|c|}
\hline Teórico conceptual & Metodológica & Práctico & Ético-político \\
\hline $\begin{array}{l}\text { Aplicación a una situación } \\
\text { real de estrategias de levan- } \\
\text { tamiento de datos CUAN- } \\
\text { CUAL revisadas solo de } \\
\text { forma teórica en asignaturas } \\
\text { previas. }\end{array}$ & $\begin{array}{l}\text { Sensibilización sobre la importan- } \\
\text { cia del diseño flexible de la IAP (en } \\
\text { términos de anticipación frente a } \\
\text { situaciones emergentes no contem- } \\
\text { pladas), lo cual no debe ser confun- } \\
\text { dido con decisiones "espontáneas" o } \\
\text { "voluntaristas". }\end{array}$ & $\begin{array}{l}\text { Fortalecimiento de } \\
\text { habilidades sociales } \\
\text { transversales, tales } \\
\text { como: a) trabajo en } \\
\text { equipo, b) escucha ac- } \\
\text { tiva, c) capacidad de } \\
\text { síntesis, d) comunica- } \\
\text { ción asertiva, e) empa- } \\
\text { tía y f) negociación. }\end{array}$ & $\begin{array}{l}\text { Concienciación ante } \\
\text { la experiencia ne- } \\
\text { gativa de la comuni- } \\
\text { dad afectada por el } \\
\text { desastre, la cual se } \\
\text { arrastra más allá de } \\
\text { la contingencia del } \\
\text { evento. }\end{array}$ \\
\hline
\end{tabular}


Problematización conceptual-empírica ante la problemática concreta abordada (ruptura tanto con el empirismo ingenuo [Afectados como meras víctimas] o el imperialismo de la teoría [Comunidades resilientes ante el desastre]).

Incorporación de procesos de autorreflexión grupales los cuales posibilitaron no solo la incorporación de las propias narrativas estudiantiles sino también el señalamiento colectivo de prejuicios u obstaculizadores del proceso.
Desarrollo y aplicación procedimental de estrategias CUAL-CUAN de acuerdo con una problemática de relevancia social.

Relevo de la importancia de la horizontalidad de la IAP en pos de desarrollar soluciones colaborativas.

Fortalecimiento de la criticidad metodológica, por ejemplo: a) Adecuación de la técnica según las características de la población objetivo; b) Pertinencia del tipo de técnica según la naturaleza del dato; b) Limitaciones del diseño y/o formato de los instrumentos según el contexto (Problemas en la comprensión de la escala Likert en adultos mayores y/o uso limitado escalas de auto aplicación en personas que no saben leer).
Aplicación de conocimientos disciplinares a través de un trabajo de campo guiado por una problemática real.
Desarrollo de un posicionamiento investigativo-reflexivo e implicativociudadano ante la problemática.
Retroalimentación comunitaria continua para incorporar estrategias/ procedimientos de acuerdo con las necesidades emergentes.
Continuidad con el trabajo comunitario post asignatura (algunos estudiantes siguieron vinculados a la comunidad).

Fuente: Elaboración propia.

Tabla 4. Limitaciones en la enseñanza-aprendizaje del diagnóstico psicosocial bajo ApS

Teórico conceptual

Abordaje superficial sobre el componente interventivo de la gestión comunitaria ante desastres, esto debido a los tiempos acotados de la asignatura. competencias profesionales enseñadas y las competencias desplegadas ante la problemática psicosocial concreta esto debido a las características de cada contexto.

\section{Metodológica}

Falta de sistematicidad y de análisis profundo de los datos levantados (tanto en términos estadísticos como de contenido cualitativo), esto tanto por los tiempos acotados de la asignatura, como por los vacíos metodológicos previos (desconocimiento en el manejo de software).

Falta de incorporación de criterios de rigurosidad y calidad de las técnicas utilizadas.

\section{Práctico}

Ético - Político

Limitación temporal, en términos de duración semestral (solo 13 sesiones) como por la cantidad de horas asignadas a estas (dos horas aprox.).

Limitación de recursos económicos (transporte y materiales-tecnológicos para el levantamiento de datos) así como humanos (tanto de los profesores como de la comunidad).

Parte de la comunidad desconfía ante el trabajo de organizaciones externas.
En algunos casos, baja disposición y motivación por parte del estudiantado, debido al interés profesional en otras áreas profesionales (ej. clínica, laboral, educacional).

Incompatibilidad, en algunos casos, entre las necesidades sentidas de la comunidad (quienes estaban más interesadas en la reconstrucción habitacional) como en las necesidades a satisfacer en la asignatura (diagnóstico psicosocial).

Fuente: Elaboración propia 


\section{Discusión y conclusiones}

En primer lugar, valoramos de forma positiva el uso de ApS en el proceso de enseñanza-aprendizaje del diagnóstico comunitario ante el riesgo de desastre, identificando tanto ventajas como limitaciones en la adquisición y desarrollo i) teórico-conceptual, ii) metodológico, iii) práctico y iv) ético-político de competencias disciplinares y transversales a nivel profesional en ciencias sociales.

En el proceso de enseñanza-aprendizaje, estas ventajas se tradujeron, no solo en el fortalecimiento práctico de habilidades, conocimientos $\mathrm{y}$ actitudes, en término de competencias profesionales (CNA, 2015; Departamento de psicología, 2015; Juliá, 2013), sino también para el relevo heurístico de distintas técnicas investigativas utilizadas para el diagnóstico. En esta línea, relevamos la importancia de ampliar los horizontes prácticos de la enseñanza convencional metodológica en contextos formativos universitarios, la cual solo tiende a cobrar sentido al momento de cursar la tesis de grado, escindiéndose de las competencias técnicas disciplinares necesarias para el desarrollo de diagnósticos e intervenciones de carácter psicosocial (Bassi, 2015; LópezNoguero, 2017; Orellana-Fonseca et al., 2019). Es por esto, que por medio de la metodología ApS logramos que ciertos conocimientos conceptuales-abstractos e investigativos enseñados en aula, fuesen traducidos y trasladados a una situación problemática concreta, posibilitando no solo el manejo procedimental de las distintas técnicas utilizadas [habilidad], sino también, un autoanálisis crítico reflexivo respecto su uso [actitud] (Cuadra-Martínez et al., 2018).

Otro elemento importante para destacar es el complemento entre estrategias activas de enseñanza y el aprendizaje aplicado y horizontal del proceso de investigación. Es así como el ApS permitió al estudiantado no solo posibilitar una aproximación situada al conocimiento profesional, sino también conocer la importancia de la horizontalidad participativa propia de la IAP tanto para i) posibilitar diálogos de saberes rea- les entre expertos y comunidades, como para ii) posicionar a estos últimos como un actor protagónico en los procesos de cambio autogestionados y sostenibles en el tiempo (Stringer, 2008). De esta forma, los estudiantes pudieron no solo integrar contextualmente teoría y práctica, además de desarrollar competencias profesionales ante problemáticas societales emergentes, sino que - además - el servicio ofertado logró sentido y utilidad para la comunidad, siendo esto un factor clave para los procesos de cambio intersubjetivo (Sandoval-Díaz et al., 2020). Esta complementariedad podría fortalecer la metodología de la ApS, disminuyendo las limitaciones que se producen cuando comunidades e interventores parten desde supuestos inconmensurables sobre el potencial servicio.

Respecto al objetivo del servicio para/ con la comunidad, se logró concretar incipientemente un círculo virtuoso entre intencionalidad pedagógica y social (Furco, 2011; Pizarro \& Hasbún, 2019), posibilitando una negociación continua entre necesidades sentidas comunitarias y los objetivos de aprendizaje esperados, teniendo como meta viable clara el diagnóstico social. Sin embargo, si bien se logró alcanzar este objetivo pedagógico en los tiempos esperados, muchas de las necesidades sociales emergentes del servicio desarrollado no pudieron ser abordadas, dado que algunas obedecían estrictamente o a la dimensión física-material de la reconstrucción de viviendas y espacio público, como a decisiones institucionales que emanaban de los gobiernos locales (estrategias de mitigación ante la exposición de riesgos (Anderson \& Woodrow, 1989). Otro aspecto pendiente fue la incorporación de estrategias interventivas para la reducción de la vulnerabilidad social territorial (Freire, 2012), así como también el empoderamiento y fortalecimiento de capacidades resistentesresilientes (Sandoval et al., 2018).

En lo práctico, en términos de limitaciones, de acuerdo con los cuadrantes del ApS del Service-learning Center de la Universidad de Stanford, nuestra experiencia pedagógica estu- 
vo más cercana a una mixtura de trabajo de campo y servicio comunitario, por la ausencia del componente de retroalimentación por parte de la comunidad, esto debido a los factores de (i) temporalidad acotada de la intervención, (ii) articulación incipiente pero no sistemática entre interventores y socios colaboradores, (ii) baja implicancia y empoderamiento territorial y iii) alta desconfianza hacia agentes externos. A estas limitaciones se suman la carencia de activos materiales y humanos adecuados para una intervención de mediana escala, así como también la ausencia de actores gubernamentales, los cuales eran centrales para las decisiones y tiempos para la reconstrucción posdesastre (Mayor, 2019). Por tanto, futuros trabajos debiesen incorporar la propuesta planteada en el marco de Acción de Sendai (2015), bajo la cual:

Las organizaciones comunitarias deben participar, en colaboración con las instituciones públicas, para, entre otras cosas, proporcionar conocimientos específicos y orientación pragmática en el contexto de elaboración y aplicación de marcos normativos, estándares y planes para la RRD. (UNDRR, 2015, p. 23)

Por lo tanto, mejorar las capacidades, requiere una transferencia de poder en beneficio de la población local reconociéndolos como actores informados, hábiles e ingeniosos (Gaillard et al., 2019). No obstante, a pesar de esta limitación, desde el punto de vista formativo profesional, esta experiencia pedagógica exploratoria posibilitó ir más allá de los enfoques convencionales centrados en el desastre, posibilitando el adentrarnos en la anticipación, respuesta y recuperación local ante riesgos climáticos, bajo la articulación pragmática de distintas estrategias investigativas-interventivas (Anderson \& Woodrow, 1989; Sandoval et al., 2018). En síntesis, afirmamos el uso de la ApS como una metodología participativa para el fortalecimiento no solo de competencias profesionales-disciplinares, sino también, transversales como el pensamiento crítico, el trabajo en equipo y la sensibilidad ciu- dadana (Muñoz-Arce et al., 2017; Ortega, 2015), esto acorde al tipo de formación universitaria integral que requieren las emergentes problemáticas psicosociales complejas (Morin, 2016).

\section{Notas}

1 Trabajo financiado por la Agencia Nacional de Investigación y Desarrollo (ANID) en el marco de la ejecución del FONDECYT de Iniciación N 11200683 "Riesgos socionaturales intensificados por el cambio climático: Análisis de la vulnerabilidad-resiliencia social a escala local" (2020 a 2023)

2 En Chile como competencias transversales se identifican las capacidades de comunicación oral y escrita, resolución de problemas, trabajo en equipo, compromiso ético, creatividad, liderazgo, entre otras (CNA, 2015 p. 5).

3. Proyecto MECESUP ULS 0601 “Mejoramiento de la formación general y especifica de los profesionales psicólogos del consorcio de las universidades del estado (CUECH) mediante el diseño e implementación de un marco curricular basado en competencias".

4. Un caso es un fenómeno (o unidad) espacialmente delimitado, observado en un solo punto del tiempo o a través de un determinado periodo de tiempo (Gerring, 2007 p. 19).

5. Utilización de distintas técnicas que se encuadran en métodos de investigación diferentes, y se combinan para analizar un mismo objeto de estudio con el objetivo de aumentar calidad investigativa.

\section{Referencias bibliográficas}

Anderson, M., \& Woodrow, P. (1989). Rising from the ashes: development strategies in times of disaster. Westview Press.

Astudillo-Pizarro, F., \& Sandoval-Díaz, J. (2019). Justicia espacial, desastres socionaturales y políticas del espacio. Dinámicas sociopolíticas frente a los aluviones y proceso de recuperación en Copiapó, Chile [Spatial Justice, Socio-Natural Disasters, and Spatial Policies: Sociopolitical Dynamics in view of the Floods and Recovery Processes in Copiapó, Chile]. Cuadernos de Geografía: Revista Colombiana de Geografía, 28(2), 303-321. https://doi.org/10.15446/rcdg.v28n2.73520

Aguilar-Idañez, M., \& Ander-Egg, E. (2001). Diagnóstico social: Conceptos y metodología. Lumen.

Barrón-Tirado, M. (2009). Docencia universitaria y competencias didácticas [Teaching at the University and educational competences]. Perfiles educativos, 31(125), 76-87. 
https://doi.org/10.22201/

iisue.24486167e.2009.125.18849

Bassi, J. (2015). Formulación de proyectos de tesis en ciencias sociales. Manual de supervivencia para estudiantes de pre-y posgrado. FACSO/ El buen aire.

Claire, M., Dintrans, P., Santander, G., \& Valenzuela, G. (2019). Aprendizaje servicio: Origen y trayectoria en Chile. Red Nacional de aprendizaje servicio Chile. En V. Pizarro \& B. Hasbún, B. (Eds.), Aprendizaje-Servicio en la Educación Superior Chilena. Ediciones CEAFEN Universidad de Chile. https://bit.ly/3ad0EWE

Cuadra-Martínez, D., Castro, P., \& Juliá, M. (2018). Tres saberes en la formación profesional por competencias: Integración de teorías subjetivas, profesionales y científicas [Three Types of Knowledge in Competency Based Professional Education: The Integration of Subjective, Professional, and Scientific Theories]. Formación universitaria, 11(5), 19-30. https://dx.doi.org/10.4067/S0718-50062018000500019

Comisión Nacional de Acreditación de Chile [CNA]. (2015, octubre). Glosario de términos complementarios criterios de acreditación de pregrado. https://bit.ly/2IQ1cGG

De Castro, A., \& Domínguez, E. (2018). Transformar para educar 6: Aprendizaje servicio. Editorial Universidad del Norte. https://bit.ly/2JXaqlb

Departamento de Psicología UDA (s.f.). Malla curricular de psicología. https://bit.ly/2Kpjv6a

Departamento de Psicología UDA (2015, Julio 9). Definición de la carrera. https://bit.ly/389LoY3

Dewey, J. (1985). Democracia y escuela. Eumo.

Fernández, J., Escalante, E., \& Richard, F. (2011). Revisitando algunas herramientas de evaluación sistémica [Revisiting some tools of systemic evaluation]. Psicoperspectivas, 10(1), 190-208. https://bit.ly/3gNkmcV

Flick, U. (2007). Introducción a la investigación cualitativa. Morata.

Flick, U. (2014). La gestión de la calidad en la investigación cualitativa. Morata.

Freire, P. (2012). Pedagogía del oprimido (2a ed.). Siglo XXI.

Furco, A. (2011). El aprendizaje-servicio: un enfoque equilibrado de la educación experiencial
[Service-Learning: A Balanced Approach to Experiential Education]. Revista Educación Global, 0, 64-70. https://bit.ly/2K01zPI

Gaillard, J., Cadag, J., \& Rampengan, M. (2019). People's capacities in facing hazards and disasters: an overview. Natural Hazards, 95(3), 863-876. https://doi.org/10.1007/s11069-018-3519-1.

García-Lirios, C., Carreón-Guillén, J., \& HernándezValdés, J. (2014). La formación profesional de capital humano en la civilización del cambio climático [Vocational training in human capital civilization of climate change]. Revista Internacional de Investigación en Ciencias Sociales. Revista Internacional de Investigación en Ciencias Sociales, 10(1), 107125. https://bit.ly/3oRTWJO

Gerring, J. (2007). What is a case study and what is it good for? American Political Science Review, 98(2), 341-354. https://doi.org/10.1017/ S0003055404001182

Griselda-Günther, M., \& Gutiérrez, R. (2017). La política del ambiente en América Latina. Una aproximación desde el cambio ambiental global. CLACSO.

Intergovernmental Panel on Climate Change [IPCC]. (2014). AR5 Climate Change 2014: Impacts, Adaptation, and Vulnerability. Cambridge University Press.

Izquierdo, T., Abad, M., Justo, B., Bernárdez., E., \& Arancibia, M. (2018). El evento hidrometeorológico del 25M en la ciudad de Copiapó: análisis de la inundación y los daños en el casco urbano. En G. Vargas, S. Pérez \& P. Aldunce (Eds.), Aluviones y resiliencia en atacama: construyendo saberes sobre riesgos $y$ desastres (pp. 117-135). Social-Ediciones.

Juliá, M. (2013). Competencias del psicólogo en Chile: Propuesta desde las universidades estatales. Editorial Universidad de La Serena.

López-Noguero, F. (2017). Metodología participativa en la enseñanza universitaria. Ediciones de la U.

Matus, T. (2002). Propuestas contemporáneas en trabajo social: hacia una intervención polifónica. Espacio Editorial

Mayor, D. (2019). Dimensiones pedagógicas que configuran las prácticas de aprendizaje-servicio [Pedagogical Dimensions that Configure 
Service-Learning Practices]. Páginas de Educación, 12(2), 23-42. https://dx.doi. org/10.22235/pe.v12i2.1834

Montes, R., Tapia, M., \& Yaber, L. (2011). Manual para docentes y estudiantes solidarios. CLAYSS. https://bit.ly/37ni8hn

Morin, E. (2016). Enseñar a vivir: Manifiesto para cambiar la educación. Paidós.

Muñoz-Arce, G., Hernández-Mary, N., \& VélizBustamante, C. (2017). La relación entre investigación e intervención social: voces desde el trabajo social chileno [The relationship between research and social intervention: voices from chilean social work]. Trabajo Social Global. Revista de Investigaciones en Intervención Social, 7(12), 3-24. https://bit. ly/3gQXmcN

Orellana-Fonseca, C., Salazar-Jiménez, R., FaríasOlavarría, F., Martínez-Labrin, S., \& PérezDíaz, G. (2019). Valoraciones que estudiantes de un posgrado de profesión docente tienen sobre la formación en metodología de la investigación recibida en el pregrado y su uso en la práctica docente [Postgraduate Teaching Students' Evaluation of the Training in Research Methodology Received During Their Degree, and its Use in Teaching Practice]. Revista Electrónica Educare, 23(1), 342-366. https://dx.doi.org/10.15359/ree.23-1.17

Ortega, M. (2015). Trabajo social como transdisciplina: hacia una teoría de la intervención [Social work as transdiscipline: towards a theory of intervention]. Cinta de moebio, (54), 278-289. https://dx.doi.org/10.4067/S0717-554X2015000300005

Pellicer, I., Vivas-Elias, P., \& Rojas, J. (2013). La observación participante y la deriva: dos técnicas móviles para el análisis de la ciudad contemporánea. El caso de Barcelona [a observación participante y la deriva: dos técnicas móviles para el análisis de la ciudad contemporánea. El caso de Barcelona]. EURE, 39(116), 119-139. https://doi.org/10.4067/S0250-71612013000100005

Pizarro, V., \& Hasbún, B. (2019). Aprendizaje Servicio en la Educación Superior Chilena. Ediciones CEA-FEN Universidad de Chile. https://doi.org/10.34720/WCC5-5184

Ricardo, D., Guerra, M., Morales, C., \& Rifa, J. (2019). La Universidad y la educación para el cambio climático [The university and the education for the climate change]. Humanidades Médicas, 19(3), 427-442. https://bit.ly/3r0JeCI

Rodríguez, M. (2014). Aprendizaje-Servicio como estrategia metodológica en la Universidad [Service-learning as a methodological strategy at University]. Revista Complutense de Educación, 25(1), 95-113. https://doi.org/10.5209/rev_RCED.2014.v25.n1.41157

Sánchez, L., \& Reyes, O. (2015, diciembre). Medidas de adaptación y mitigación frente al cambio climático en América Latina y el Caribe. Una revisión general. Estudios del Cambio Climático en América Latina. CEPAL. https://bit.ly/3aejY5O

Sandoval-Díaz, J. (2020). Vulnerabilidad-resiliencia ante el proceso de riesgo-desastre: Un análisis desde la ecología política [Vulnerabilityresilience to the risk-disaster process: An analysis from the political ecology]. Polis (Santiago), 19(56), 214-239. https://dx.doi.org/10.32735/s0718-6568/2020-n56-1527 Sandoval-Díaz, J., \& Cuadra-Martínez, D. (2020). Vulnerabilidad social, severidad subjetiva y crecimiento postraumático en grupos afectados por un desastre climatológico [Social vulnerability, subjective severity and posttraumatic growth in groups affected by a climate disaster]. Revista de Psicología, 29(1), $1-15$. https://dx.doi.org/10.5354/0719-0581.2020.58002 Sandoval-Díaz, J., Cuadra-Martínez, D., PalaciosDíaz, D., \& Ligueño-Espinoza, S. (2020). La investigación-acción como promotora de la formación ciudadana en/desde la escuela: tres propuestas investigativas-interventivas. En C. Orellana., R. Salazar \& V. Hasse (Eds.), Formación ciudadana en la escuela: conceptualización, herramientas de intervención socioeducativa y propuestas didácticas (pp. 173-191). Ril Editores.

Sandoval., J, Rojas, L., Villalobos, M., Sandoval, C., Moraga, F., \& Aguirre, N. (2018). De organización vecinal hacia la gestión local del riesgo: diagnóstico de vulnerabilidad y capacidad [From the local organization towards local risk management: diagnosis of vulnerability and capacity]. Revista INVI, 33(92), 155-180. 
http://dx.doi.org/10.4067/S0718-83582018000100155

Stringer, E. (2008). Action research in education (2nd ed.). Pearson Prentice Hall.

Vélez, I., Rátiva, S., \& Varela, D. (2012). Cartografía social como metodología participativa y colaborativa de investigación en el territorio afrodescendiente de la cuenca alta del río Cauca [Social Cartography as a Participative and Collaborative Research Methodology in the Upper Basin of the Cauca River]. Cuadernos de Geografía, 21(2), 59-73. https://doi.org/10.15446/rcdg.v21n2.25774

Wendler, R. (2012). Human subject's protection: A source for ethical service-learning practi- ce. Michigan Journal of Community Service Learning, 2(18), 29-39.

https://bit.ly/34dhQY8

UN Office for Disaster Risk Reduction [UNDRR]. (2015). Sendai Framework for Disaster Risk Reduction 2015-2030. UNDRR. https://bit.ly/3oS2TCU

UN Office for Disaster Risk Reduction (2019 enero 23). 2018: 60 millones de personas resultaron afectadas por diversos eventos meteorológicos extremos. https://bit.ly/3oUa1hZ

United Nations Educational, Scientific, and Cultural Organization [UNESCO]. (2015). Not just hot air: Putting climate change education into practice. UNESCO https://bit.ly/3nr7LP2 